\title{
Incidence and risk factors of immune reconstitution inflammatory syndrome in HIV-TB coinfected patients
}

\begin{abstract}
Tuberculosis is one of the leading causes of development of Immune reconstitution inflammatory syndrome (IRIS) in HIV patients receiving antiretroviral therapy (ART). Objective: To determine the incidence of IRIS in HIV-TB coinfected patients, and to find out the possible risk factors associated with IRIS. Materials and Methods: Study commenced with 96 patients adhered to standard antitubercular therapy (ATT) and ART without defaultering, and followed up for six months. Result: The mean $( \pm$ SD) CD4 count and CD4 percentage at baseline was $59.16( \pm 24.63)$ per $\mathrm{mm}^{3}$ and $4.59 \%( \pm 1.73)$ respectively. Only $18.75 \%$ developed IRIS after 57.05 ( \pm 14.12$)$ days of initiation of ART. Extrapulmonary tuberculosis was the most significant factor associated with IRIS $(83.33 \%)$ than those without IRIS $(44.87 \%)(\mathrm{p}=0.0032)$. Specifically, tubercular lymphadenitis $(38.88 \%, \mathrm{p}=0.0364)$ and disseminated tuberculosis $(33.33 \%$, $\mathrm{p}=0.0217)$ were significantly associated with IRIS. The other risk factors associated with appearance of IRIS were higher CD4 count $(\mathrm{p}=0.0212)$ at three months after initiation of ART and increment of CD4 count $(\mathrm{p}=0.0063)$ and CD4 percentage $(\mathrm{p}=0.0016)$ during this period. The major manifestations of IRIS were fever (40\%), followed by lymphadenitis (38\%). The mortality rate in IRIS was not higher than those without IRIS. Conclusion: Patients with extrapulmonary tuberculosis, especially tubercular lymphadenitis, were more likely to develop IRIS and fever was associated in most of them. Higher increment of CD4 count may indicate development of IRIS in presence of new or worsening tuberculosis lesion.
\end{abstract}

Keywords: HIV; tuberculosis; immune reconstitution inflammatory syndrome; CD4 lymphocyte count.

\section{INTRODUCTION}

Human immunodeficiency virus (HIV) infection today is one of the major causes of mortality and morbidity globally. Association of tuberculosis (TB) coinfection with HIV poses greater therapeutic challenge. In developing nations, patients often present late with advanced HIV infection along with different opportunistic infections, most commonly tuberculosis. ${ }^{1}$ Tuberculosis in HIV-infected patients often presents with diagnostic difficulties, with increased chance of negative sputum smear results, atypical chest $\mathrm{X}$-ray findings, negative tuberculin skin test, lack of classical granuloma formation in late stage and also presence of more frequent extrapulmonary tuberculosis. With the advent of highly active antiretroviral therapy (HAART), incidence rate of immune reconstitution inflammatory syndrome
(IRIS) also raised significantly. Classically IRIS is defined as "occurrence or manifestation of new or existing opportunistic infection within six weeks to six months after initiation of antiretroviral therapy (ART), with associated increase in CD4 count". 2 Any opportunistic infection may present with IRIS though tuberculosis (TB) is the major cause. Other important manifestations include $\mathrm{CMV}$ retinitis, Pneumocystis jiroveci pneumonia, herpes simplex infection, varicella zoster virus infection, cryptococcal meningitis, hepatitis $B$, hepatitis $C$ infection and even some auto-immune diseases. ${ }^{3}$ The main features of IRIS with TB are increasing pneumonitis, development of pleural effusion, occurrence of new radiographic lesions, painful and enlarged lymphadenopathy, often with discharging sinus, persistent fever, development of meningitis, expanding intracranial
Authors

Dibyendu De

Rathindra Nath Sarkar ${ }^{2}$

Sibaji Phaujdar ${ }^{1}$

Kuntal Bhattacharyya ${ }^{1}$

Hare Krishna Pal ${ }^{1}$

${ }^{1}$ MBBS; Postgraduate

Trainees, Department of

Medicine, Medical College,

Kolkata, India

${ }^{2} \mathrm{MSc}$ in Medicine;

Professor, Department of

Medicine, Medical College,

Kolkata, India

Submitted on: 05/03/2011 Approved on: 07/17/2011

Correpondence to:

Dibyendu De

Tentulberia, Garia,

Kolkata

West Bengal

India, 700084

de.dibyendu@gmail.com

We declare no conflict of interest.

(C2011 Elsevier Editora Ltda. All rights reserved. 
space occupying lesions etc., ${ }^{4,5}$ It is hypothesized that pro-inflammatory cytokines produced excessively in response to systemic bacterial lipopolysacharide, nonspecifically act on latent mycobacterial antigens leading to clinical deterioration and paradoxical worsening of inflammatory responses. ${ }^{6}$ In most of the cases, IRIS regress spontaneously without any significant mortality but sometimes it may cause worsening clinical scenario with respiratory failure and tracheal compression by enlarged lymph nodes resulting in death. It should be differentiated from exacerbation of tuberculosis due to drug resistance or non-compliance, appearance of new tubercular lesion or other opportunistic infection due to advancement of HIV. Till date, there is lack of clinical data about occurrence of IRIS in HIV and TB coinfected patients in Indian population. Therefore, we conducted a cross-sectional study in a tertiary care set-up to find out prevalence and risk factors of IRIS in HIV-TB coinfected patients.

\section{MATERIAL AND METHODS}

Recently diagnosed HAART naive HIV-infected patients, who had clinical, radiological or microbiological evidence of active TB, were selected for our study. Inclusion criteria were as follows:

Recently diagnosed HIV positive by ELISA method. (According to NACO guideline, a symptomatic suspected HIV-infected person is diagnosed HIV positive when the serum sample is reactive with at least two out of three different ELISA kit). ${ }^{2}$

Patient having active tuberculosis diagnosed by clinical features, radiological appearances, presence of granuloma in aspiration material, or microscopy and/or culture positive acid-fast bacilli (AFB).

Receiving antitubercular therapy (ATT) from Revised National Tuberculosis Control Programme (RNTCP).

Started ART after 15 days of ATT as per protocol followed in the institution.

All the patients were followed up for the period of six months. The patients with non-compliance with either ATT or ART during the follow-up period were excluded from the study. The diagnosis of IRIS was done using the pre-set criteria as follows:

The patient had to be on ART with rising CD4 count.

Prior to starting ART, the patient must have documented radiological, histological or microbiologically confirmed tuberculosis.

Prior to starting ART, patient must have improvement of tuberculosis by antitubercular therapy.

Following initiation of ART, the patients must have appearance of new symptoms or re-appearance of previous symptoms.

The patients must have spontaneous resolution of symptoms with continuation of same treatment.
Our primary objective was to determine the incidence of IRIS in HIV-TB coinfected patients. Secondarily we tried to find out the possible risk factors for emergence of IRIS. The patients were divided into two groups according to development of IRIS or not. The demographic, clinical features, site of tuberculosis, presence of other opportunistic infection, baseline and follow-up CD4 count were measured and compared between the two groups. The statistical analysis was done, using chi-square test and Student's $t$ test using SPSS ver- 16 to determine the significance of the variables. A p-value $<0.05$ was taken to be statistically significant. A total of 96 patients, who were attending Medicine outdoor and indoor of Medical College Kolkata from May 2009 to April 2010, who met the eligibility criteria, were included in this study. The study was approved by the institutional ethical committee. The necessary informed consent was taken from all the patients.

\section{RESULTS}

Out of 96 patients suffering from both HIV and tuberculosis, $84(87.5 \%)$ of them male, only $18(18.75 \%)$ patients developed IRIS (Table 1$)$. The initial mean $( \pm$ SD) CD4 count of these patients was $59.16( \pm 24.63)$ per $\mathrm{mm}^{3}$ and initial mean CD4 percentage was $4.59 \%( \pm 1.73)$. The baseline parameters of the patients developing IRIS and those who did not develop IRIS were compared (Table 1). The male:female ratios of both groups were comparable. There was no significant difference between the two groups in respect of mode of transmission of HIV. But the distribution of tuberculosis is strikingly different between the two groups. Those, who developed IRIS had significantly higher incidence of extrapulmonary tuberculosis $(83.33 \%)$ than those who did not develop IRIS (44.87\%) $(\mathrm{p}=0.0032)$. Of the various forms of extrapulmonary tuberculosis in IRIS positive patients, most had tubercular lymphadenitis $(38.88 \%)$. None of the IRIS positive patient had CNS tuberculosis. The initial CD4 count in both groups were comparable, but the patients with IRIS experienced significantly higher increments of mean CD4 count $\left(45.22 \pm 18.17\right.$ per $\left.\mathrm{mm}^{3}\right)$ than those without IRIS $\left(31.37 \pm 14.88\right.$ per $\left.\mathrm{mm}^{3}\right)$, and this was statistically significant $(\mathrm{p}=0.0063)$ (Table 1). Also after three months, the mean CD4 count in patients with IRIS $\left(105.56 \pm 23.76\right.$ per $\left.\mathrm{mm}^{3}\right)$ were significantly higher than those without IRIS $(90.26 \pm 24.23$ per $\left.\mathrm{mm}^{3}\right)(\mathrm{p}=0.0212)$. The symptoms during development of IRIS are described in Table 2 and Table 3. Among the various systemic symptoms developed with IRIS, fever was predominant involving $40 \%$ patients of IRIS. The second most common symptom was generalized lymphadenopathy $(38 \%)$. The incidence of flare up of pulmonary tuberculosis was $18 \%$, while that of abdominal tuberculosis manifested by tubercular ascites and enlargement of abdominal lymph nodes was 27 percent. Only five patients required hospital admission for the severe manifestation of IRIS and intra-venous hydrocortisone was administered as a life-saving measure. Two of them died 
Table 1. Baseline characteristics of all HIV-TB coinfected patients

\begin{tabular}{|c|c|c|c|c|}
\hline & $\begin{array}{l}\text { IRIS pos } \\
(n=18)\end{array}$ & $\begin{array}{l}\text { Iris neg } \\
(\mathrm{n}=78)\end{array}$ & p value & $\begin{array}{c}\text { Total } \\
(n=96)\end{array}$ \\
\hline \multicolumn{5}{|l|}{ Sex } \\
\hline Male & $16(88.88 \%)$ & $68(87.18 \%)$ & 0.8433 & $84(87.5 \%)$ \\
\hline Female & 2 (11.12\%) & 10 (12.82\%) & & 12 (12.5\%) \\
\hline \multicolumn{5}{|l|}{ Mode of infection } \\
\hline Sexual & 16 (88.88\%) & 72 (92.31\%) & 0.6362 & 88 (91.67\%) \\
\hline Blood transfusion & $2(11.12 \%)$ & $6(7.69 \%)$ & & $8(8.33 \%)$ \\
\hline \multicolumn{5}{|l|}{ Site of tuberculosis } \\
\hline Pulmonary TB & $3(16.66 \%)$ & 43 (55.13\%) & 0.0032 & 46 (47.92 \%) \\
\hline Extrapulmonary TB & 15 (83.33\%) & 35 (44.87\%) & 0.0032 & $50(52.08 \%)$ \\
\hline TB lymphadenitis & 7 (38.88\%) & $13(16.67 \%)$ & 0.0364 & 20 (20.83\%) \\
\hline Abdominal TB & $2(11.12 \%)$ & $8(10.26 \%)$ & 0.5374 & $10(10.42 \%)$ \\
\hline CNS TB & $0(0 \%)$ & $5(6.41 \%)$ & 0.8926 & 5 (5.21\%) \\
\hline Disseminated TB & $6(33.33 \%)$ & 9 (11.54\%) & 0.0217 & $15(15.625 \%)$ \\
\hline \multicolumn{5}{|l|}{ CD4 counts } \\
\hline CD4 baseline & $60.33 \pm 24.13$ & $58.88 \pm 24.89$ & 0.8213 & $59.16 \pm 24.63$ \\
\hline CD4 \% baseline & $4.59 \pm 1.64$ & $4.59 \pm 1.76$ & 0.9938 & $4.59 \pm 1.73$ \\
\hline CD4 after 3 months & $105.56 \pm 23.76$ & $90.26 \pm 24.23$ & 0.0212 & $93.13 \pm 24.76$ \\
\hline CD4 \% after 3 months & $8.67 \pm 2.28$ & $7.01 \pm 1.99$ & 0.0091 & $7.33 \pm 2.14$ \\
\hline CD4 increments & $45.22 \pm 18.17$ & $31.37 \pm 14.88$ & 0.0063 & $33.97 \pm 16.37$ \\
\hline CD4 \% increments & $4.08 \pm 1.85$ & $2.42 \pm 1.20$ & 0.0016 & $2.73 \pm 1.49$ \\
\hline Death rate & 2 (11.11\%) & 7 (8.97\%) & 0.3896 & $9(9.375 \%)$ \\
\hline
\end{tabular}

Table 2. Distribution of symptoms in the patients of TB-IRIS

\begin{tabular}{lc} 
Symptoms during development of IRIS & Frequency \\
\hline Fever & $7(38.89 \%)$ \\
Fever with cough \& expectoration & $1(5.56 \%)$ \\
Fever with cough \& haemoptysis & $1(5.56 \%)$ \\
Fever with ascites & $2(11.11 \%)$ \\
Fever with lymph node enlargement & $2(11.11 \%)$ \\
\multicolumn{1}{|c}{ Fever only } & $1(5.56 \%)$ \\
\hline Cough \& expectoration only & $1(5.56 \%)$ \\
\hline Abdominal lymphadenopathy only & $3(16.67 \%)$ \\
\hline Lymph node enlargement only & $7(38.89 \%)$ \\
\hline Total & 18 \\
\hline Mean duration of development of IRIS & $57.05 \pm 14.12$ \\
\hline
\end{tabular}

in hospital. ART was continued in all of the patients. The patients who did not develop IRIS mostly improved clinically with resolution of their symptoms. Only 11 of 78 non-IRIS patients (14.1\%) had clinical deterioration, of which three had pulmonary tuberculosis, and the others had extrapulmonary tuberculosis. These patients did not show any initial improvement. The mortality rates in both TB-IRIS and non-IRIS groups were comparable. The cause of death in one of the two persons suffering from IRIS was the compression of trachea by enlarged cervical lymph nodes, while the other died due to disseminated tuberculosis leading to multi-organ failure. Out of seven non-IRIS patients who died during the study, three had CNS tuberculosis, mostly meningitis, and four had disseminated tuberculosis. None of the patient with pulmonary tuberculosis died during the study. The mean duration of development of IRIS after initiation of ART was 57.05 ( \pm 14.12) days (Table 2$)$. The ART followed was according to the NACO guideline and was similar in both groups. ART was started in all patients after 15 days of ATT. 


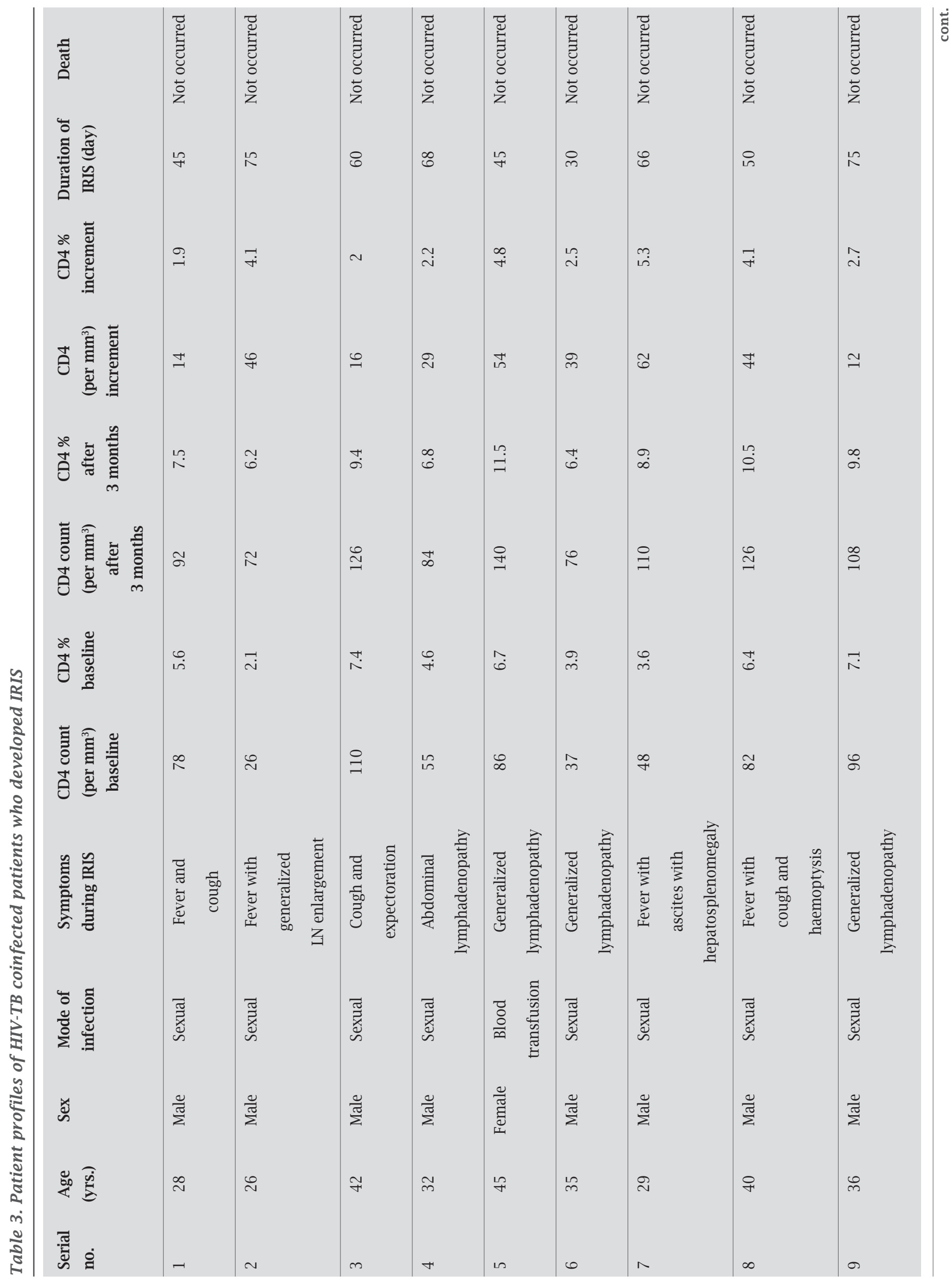




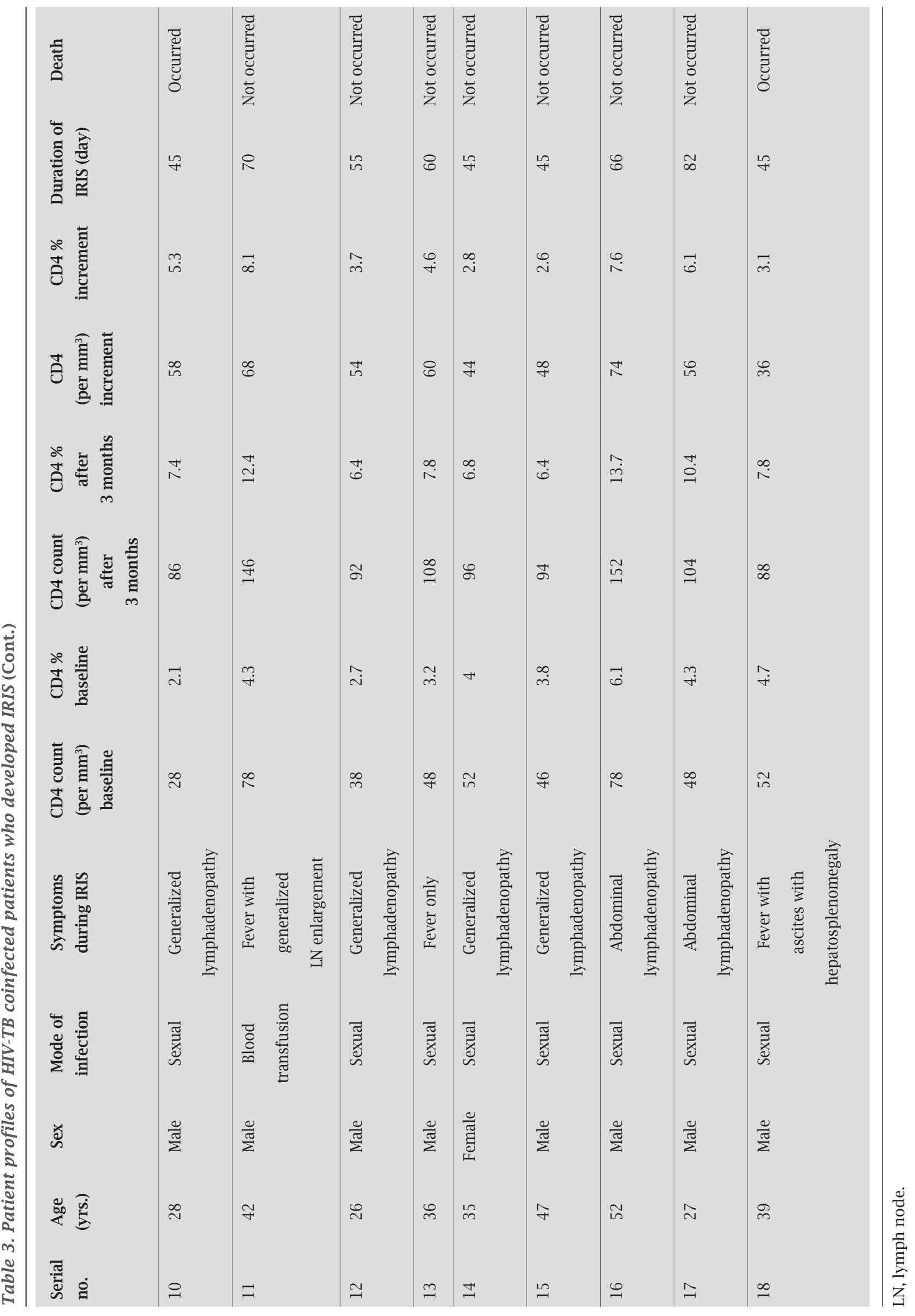




\section{DISCUSSION}

The incidence of IRIS varies throughout the world. In preHAART era, incidence of IRIS in developed countries was reported to be about $36 \% .^{7}$ In developing countries, due to higher prevalence of tuberculosis, the incidence of IRIS is also higher, though remains largely under reported. The incidence in developing countries ranges from $11 \%$ to $43 \%$, whereas in India it was reported as $8 \%{ }^{6,8}$ In our study, incidence of TB-IRIS was $18.75 \%$. As HAART is now widely available in India, the incidence of IRIS is likely to be increasing. The IRIS appears mostly within first three months of initiating ART. In our study the mean duration of appearance of IRIS was 57.05 days. The early appearance of IRIS may be due to early initiation of ART, after 15 days of ATT, in all patients. Other studies from developing countries like Brazil also documented similar result. ${ }^{9}$ Shelburne et al. ${ }^{10}$ showed that IRIS is more likely to develop earlier if HAART is initiated earlier. Most of the IRIS patients experienced an exacerbation of extrapulmonary tuberculosis, tubercular lymphadenitis being most common. Significantly $27 \%$ of the patients presented with intra-abdominal flaring up of tuberculosis. Various previous studies also showed similar result though occurrence of intra- abdominal tuberculosis was not high. ${ }^{11-13}$ This highlights that one must look out for the enlargement of abdominal lymph nodes as a marker of IRIS. Interestingly, in our study, none had CNS tubercular manifestation. This may be because CNS involvement in TB-IRIS usually occurs late with median interval being 5-10 months. ${ }^{14}$ The risk factor emerged from the study was presence of extrapulmonary tuberculosis, of which tubercular lymphadenitis and disseminated tuberculosis predominates. These findings corroborates with the findings of previous western studies, though they did not show disseminated tuberculosis as a risk factor. ${ }^{15}$ This may be due to the fact, that exposure to high load of mycobacterium and their interaction with the immune system in disseminated form of tuberculosis increases the risk of IRIS. ${ }^{16}$ The baseline CD4 count was not a risk factor for emergence of IRIS. Although previous studies, including those from different developing countries with high prevalence of HIV, postulated that CD4 counts less than 100 per $\mathrm{mm}^{3}$ are associated with increased incidence of IRIS, this was not present in our study., ${ }^{9,17}$ Rather, the increase in absolute as well as percentage of CD4 count was significantly higher in IRIS group of patients than the other. Similar result was shown by Breton $\mathrm{G}$ et al. ${ }^{12}$ This may be due to the inherent pathophysiology of IRIS development. With the initiation of ART, there is partial restoration of immune response, which causes increase in CD4 count. As IRIS occurs due to excessive effect of various inflammatory mediators liberated from activated lymphocytes, so increment of CD4 count is related with occurrence of IRIS and it may act as a surrogate marker of development of IRIS, though more detailed studies need to be done to know the actual predictive value. The major limitation of the study was exclusion of subclinical tuberculosis patients who may develop IRIS after initiation of ART. Also all the patients were given standard ATT and ART, so effect of variation of therapy with emergence of IRIS was not evaluated. In addition, because ART was started after 15 days of ATT in all patients, any effect of delay of ART with appearance of IRIS could not be studied. All the patients recruited in the study were in advanced HIV. Further study is needed to know the variation of incidence of IRIS at different stages of HIV. Although most of our patients recovered spontaneously without requiring any deviation from their treatment protocol, large prospective study will guide us about proper management of IRIS.

\section{CONCLUSION}

In conclusion, IRIS in HIV-TB coinfected patients is an important entity, $12 \%$ of the patients developed IRIS in our study within two months of starting ART. Patients with extrapulmonary tuberculosis, especially tubercular lymphadenitis are more prone to develop IRIS. Fever is associated in most of these patients. Higher increment of CD4 count may give warning for development of IRIS in presence of new or worsening tuberculosis lesion.

\section{REFERENCES}

1. John M, French MA. Exacerbation of the inflammatory response to Mycobacterium tuberculosis after antiretroviral therapy. Med J Aust. 1998; 169(9):473-4.

2. Antiretroviral Therapy Guidelines for HIV-infected Adults and adolescents including post-exposure prophylaxis by NACO 2007

3. Murdoch DM, Venter WD, Van Rie A, Feldman C. Immune reconstitution inflammatory syndrome (IRIS): review of common infectious manifestations and treatment options. AIDS Res Ther. 2007; 4:9.

4. Lawn SD, Bekker LG, Miller RF. Immune reconstitution disease associated with mycobacterial infections in HIV-infected individuals receiving antiretrovirals. Lancet Infect Dis. 2005; 5(6):361-73.

5. Sungkanuparph S, Vibhagool A, Mootsikapun P, Chetchotisakd P, Tansuphaswaswadikul S, Bowonwatanuwong C. Opportunistic infections after the initiation of highly active antiretroviral therapy in advanced AIDS patients in an area with a high prevalence of tuberculosis. AIDS. 2003; 17(14):2129-31.

6. Shankar EM, Vignesh R, Murugavel KG, et al. Immune reconstitution inflammatory syndrome in association with HIV/AIDS and tuberculosis: views over hidden possibilities. AIDS Res Ther. 2007; 4:29-42.

7. Narita M, Ashkin D, Hollender ES, Pitchenik AE. Paradoxical worsening of tuberculosis following antiretroviral therapy in patients with AIDS. Am J Respir Crit Care Med. 1998; 158(1):157-61.

8. Gazzard B; BHIVA Writing Committee. British HIV Association (BHIVA) guidelines for the treatment of HIV-infected adults with antiretroviral therapy (2005). HIV Med. 2005; 6 Suppl 2:1-61. 
9. Serra FC, Hadad D, Orofino RL, et al. Immune reconstitution syndrome in patients treated for HIV and tuberculosis in Rio de Janeiro. Braz J Infect Dis. 2007; 11(5): 462-65.

10. Shelburne SA, Visnegarwala F, Darcourt J, et al. Incidence and risk factors for immune reconstitution inflammatory syndrome during highly active antiretroviral therapy. AIDS. 2005; 19(4):399-406.

11. Furrer H, Malinverni R. Systemic inflammatory reaction after starting highly active antiretroviral therapy in AIDS patients treated for extrapulmonary tuberculosis. Am J Med. 1999; 106(3):371-2.

12. Breton G, Duval X, Estellat C, et al. Determinants of immune reconstitution inflammatory syndrome in HIV type 1-infected patients with tuberculosis after initiation of antiretroviral therapy. Clin Infect Dis. 2004; 39(11):1709-12.

13. Manosuthi W, Kiertiburanakul S, Phoorisri T, Sungkanuparph $\mathrm{S}$. Immune reconstitution inflammatory syndrome of tuberculosis among HIV-infected patients receiving antituberculous and antiretroviral therapy. J Infect. 2006; 53 (6):357-63.
14. Crump JA, Tyrer MJ, Lloyd-Owen SJ, Han LY, Lipman MC, Johnson MA. Military tuberculosis with paradoxical expansion of intracranial tuberculomas complicating human immunodeficiency virus infection in a patient receiving highly active antiretroviral therapy. Clin Infect Dis. 1998; 26(4):1008-9.

15. Wendel KA, Alwood KS, Gachuhi R, Chaisson RE, Bishai WR, Sterling TR. Paradoxical worsening of tuberculosis in HIVinfected persons. Chest. 2001; 120(1):193-7.

16. Jon F. Tuberculosis. In: William P, editor. Infectious Diseases. Vol. 1. Spain: Mosby; 2004. p. 401-18.

17. Michailidis C, Pozniak AL, Mandalia S, Basnayake S, Nelson MR, Gazzard BG. Clinical characteristics of IRIS syndrome in patients with HIV and tuberculosis. Antivir Ther. 2005; 10(3):417-22. 